\title{
The Intermediolateral Cell Column of the Thoracic Spinal Cord Is Comprised of Target-Specific Subnuclei: Evidence from Retrograde Transport Studies and Immunohistochemistry
}

\author{
Nathan M. Appel and Robert P. Elde \\ Department of Cell Biology and Neuroanatomy, University of Minnesota, Minneapolis, Minnesota 55455
}

In this study we examined the hypothesis that the intermediolateral cell column (IML) of the thoracic spinal cord, the nucleus from which preganglionic sympathetic neurons originate, provides an anatomical substrate through which selective regulation of sympathetic nervous system targets is accomplished.

Preganglionic sympathetic neurons of rats were retrogradely labeled by the simultaneous exposure of the cervical sympathetic trunk (CST) and the adrenal medulla to FluoroGold and True blue, contrasting fluorescent dyes. Retrograde labeling from these sites revealed 2 populations of sympathetic preganglionic neurons in IML whose distribution overlapped between segments T1 and T4. In regions where these $\mathbf{2}$ groups of retrogradely labeled neurons overlapped, sympathoadrenal preganglionic (SAP) neurons occupied the most lateral aspect of the nucleus. It was also determined whether individual retrogradely labeled neurons within these two groups sent axon collaterals to both the CST and adrenal medulla. Diamidino yellow, a fluorescent retrograde tracer dye that labels only nuclei, was substituted for Fluoro-Gold and used in combination with True blue to simultaneously label preganglionic sympathetic neurons projecting to either the CST or adrenal medulla. No double-labeled cell bodies were observed in spinal cords of rats treated in this manner. Thus it appeared that the efferent projections of these 2 cell populations in IML were target-specific.

Immunohistochemical analysis of the relationship between nerve fibers in the IML and preganglionic sympathetic neurons was also undertaken in an attempt to classify further these 2 populations of sympathetic preganglionic neurons. Equal proportions of identified CST and SAP neurons appeared to be apposed by varicosities immunoreactive for either somatostatin or serotonin. On the other hand, when the comparison was based on whether oxytocin-immunoreactive varicosities appeared to appose these 2 populations of retrogradely labeled sympathetic neurons, a highly significant difference was revealed. That is, oxytocin-immunoreactive fibers and terminals appeared to avoid SAP neurons.

\footnotetext{
Received July 7, 1987; revised Sept. 16, 1987; accepted Sept. 26, 1987.

This investigation was supported by National Institute on Drug Abuse Grants DA05275 to N.M.A. and DA02148 to R.P.E. and INCSTAR Corporation. We wish to thank Drs. Virginia S. Seybold and Martin W. Wessendorf for their helpful comments and editorial assistance, Dr. Larry Schmued for his generous gift of Fluoro-Gold, and Mr. Gerald Sedgewick for printing the photographs.

Correspondence should be addressed to Dr. Nathan M. Appel, Ph.D., Department of Cell Biology and Neuroanatomy, University of Minnesota, 321 Church St. S.E., Minneapolis, MN 55455.

Copyright (C) 1988 Society for Neuroscience $0270-6474 / 88 / 051767-09 \$ 02.00 / 0$
}

Thus these data support the hypothesis that an anatomical substrate exists in spinal cord IML whereby selective regulation of sympathetic nervous system targets may be mediated. Moreover, the lack of oxytocin-immunoreactive varicosities apposing SAP neurons in IML suggests that if the paraventricular nucleus innervates SAP neurons in IML, it does so via a population of neurons that do not use oxytocin as a neurotransmitter.

The sympathetic division of the autonomic nervous system appears to be organized in a hierarchical manner. That is, supraspinal and spinal neurons project to preganglionic sympathetic neurons, preganglionic sympathetic neurons project to postganglionic neurons residing in the various sympathetic ganglia or to the adrenal medulla, and postganglionic sympathetic neurons project to a variety of peripheral targets. Early investigators believed that the entire sympathetic outflow changed its activity as a unit (Cannon and Rosenblueth, 1937). However, evidence has accumulated that suggests that different portions of the sympathetic outflow may be regulated independently of one another. For example, different stimuli can result in simultaneous activation of one sympathetic response and inhibition of another (Delius et al., 1972; Okada and Ninomiya, 1983). Similarly, using plasma catecholamine secretion as an index of sympathetic nervous system activity, it has been demonstrated that focal electrical stimulation of discrete but adjacent brain sites has different effects on norepinephrine than on epinephrine secretion (Stoddard-Apter et al., 1983). That is, postganglionic sympathetic nerve endings, from which the bulk of circulating norepinephrine is derived, and the adrenal medulla, from which the bulk of circulating epinephrine is derived (Price, 1957; Yamaguchi and Kopin, 1979), may respond independently of each other to a single stimulus. Consistent with this observation, others reported that intracerebral somatostatin administration inhibits stimulated epinephrine, but not norepinephrine secretion, in rats when administered simultaneously with a number of peptides (Fisher and Brown, 1980; Van Loon et al., 1981; Brown and Fisher, 1984).

For independent regulation of specific peripheral targets by the sympathetic nervous system to occur, certain minimum criteria must be met. They are that (1) discrete populations of preganglionic sympathetic neurons must innervate discrete populations of postganglionic neurons, and (2) discrete populations of neurons from "other brain centers" must innervate discrete populations of sympathetic preganglionic neurons. In the mammal, the somata of preganglionic sympathetic neurons reside in the intermediolateral cell column (IML) of the spinal cord, and their axons project to the various sympathetic ganglia and the adrenal medulla (Appenzeller, 1982). Previous studies in the 
guinea pig revealed that preganglionic sympathetic neurons projecting to different sympathetic ganglia were concentrated at specific, but sometimes overlapping, rostral/caudal levels of IML (Dalsgaard and Elfvin, 1979, 1981). However, since only a single retrograde transport marker was used to label cell popuations in these studies, it was not possible to determine whether individual preganglionic neurons, by means of axon collaterals, projected to more than one target. Subsequently Hancock (1982), using horseradish peroxidase and 4,6-diamidino-2-phenylindole (DAPI), accomplished simultaneous retrograde tract tracing from the central cut ends of the intermesenteric trunk and caudal lumbar sympathetic trunk in golden hamsters. Two distinct subgroups of retrogradely labclcd prcganglionic ncurons were revealed. They were similarly distributed between spinal segments T1 2 and L3, but no double-labeled neurons were apparent; that is, single neurons did not appear to project collaterals to both targets. Thus it was suggested that the IML could provide an anatomic substrate through which independent stimulation of different sympathetic targets is mediated.

On the basis of the above reports of nonparallel calecholamine secretion in response to various stimuli and anatomical data, it was hypothesized that the IML may provide an anatomical substrate through which independent activation of postganglionic sympathetic nerve terminals and the adrenal medulla is mediated. This hypothesis was examined by employing contrasting fluorescent retrograde dyes to label simultaneously, from peripheral sites, 2 populations of preganglionic sympathetic neurons in IML. One population was retrogradely labeled from neurons that project through the central sympathetic trunk (CST) to the superior cervical ganglion and the other from the adrenal medulla.

There is also evidence in the literature suggesting that different neurotransmitters may be responsible for the differential activation of sympathetic outflow. Simultaneous central nervous system administration of somatostatin or a somatostatin analog with a variety of neuropeptides selectively depresses stimulated epinephrine secretion, but has little effect on concomitant norepinephrine secretion (Fisher and Brown, 1980; Van Loon et al., 1981; Brown and Fisher, 1984). Moreover, in rat it was reported that somatostatin-immunoreactive fibers and terminals frequently appeared to appose retrogradely labeled sympathoadrenal preganglionic (SAP) neurons in IML. Conversely, it also has been suggested that oxytocin-immunoreactive afferents specifically avoided SAP neurons (Holets and Elde, 1982). Similarly, in cat, neither somatostatin- nor oxytocin-immunoreactive fibers and terminals were uniformly distributed in IML (Krukoff et al., 1985).

In view of these data, it was hypothesized that the neurochemical identity of afferents to SAP neurons may be different than that of preganglionic neurons projecting to a sympathetic ganglion. To examine this hypothesis, fluorescence immunohislochemistry was employed in combination with fluorescent retrograde labeling techniques to compare the chemically coded afferent input to SAP neurons with that of preganglionic sympathetic neurons projecting to the superior cervical ganglion.

Portions of these data were presented in preliminary form at the 1986 Society for Neuroscience Annual Meeting, Washington, D.C.

\section{Materials and Methods}

Male Sprague-Dawley rats, $112 \pm 6 \mathrm{gm}$ (mcan \pm SEM) (Bio-Lab, St. Paul, MN), were used in this study. While rats were under Equithesin anesthesia, SAP neurons were retrogradely labeled from the adrenal medulla with aqueous $3 \%$ (wt/vol) solutions of either True blue (Dr. Illing KG, Gross Umstadt, FRG) or Fluoro-Gold (Fluorochrome, Englewood, CO) as previously described (Appel et al., 1986; Schmued and Fallon, 1986). Preganglionic sympathetic neurons were also retrogradely labeled from axons projecting through the CST to the superior cervical ganglion (Rando et al., 1981) by dipping the cut central end of a CST for $15 \mathrm{~min}$ in aqueous 3\% (wt/vol) solutions of either True blue or Fluoro-Gold. In addition, in some rats one retrograde tracer dye was applied to one site and the wiher dye to the other sitc. In this manner, preganglionic sympathetic neurons were retrogradely labeled simultaneously from both the CST and the adrenal medulla in the same rat. Using this same methodology, some rats were also treated with True blue in combination with the fluorescent retrograde tracer dye Diamidino yellow (Dr. Illing KG). When True blue was applied to the cut CSI, Diamidino yellow ( $5 \mu \mathrm{l} ; 3 \% \mathrm{wt} / \mathrm{vol}$ in distilled $\mathrm{H}_{2} \mathrm{O}$ ) was administered via the adrenal medulla and vice versa. Seven to ten $\mathrm{d}$ following application of the retrograde tracer dyes, the rats were reanesthetized and perfused transcardially with $200 \mathrm{ml}$ of calcium-free Tyrode's solution followed by $700 \mathrm{ml}$ of $\mathrm{pH} 7.3 \mathrm{picric}$ acid/paraformaldehyde fixative (Stephanini et al., 1967). Following perfusion, identified spinal cord segments were removed and sectioned in the horizontal plane (10 $\mu \mathrm{m}$ ) as previously described (Appel et al., 1986). They were examined and photographed uncoverslipped through a Zeiss standard microscope (Carl Zeiss, Oberkochen, FRG) using transmitted dark-field UV illumination.

Some sections in which retrogradely labeled preganglionic sympathetic neurons were visible were subsequently processed for simultaneous 2-color fluorescence immunohistochemistry, using a variation of the techniques of Erichsen et al. (1982) and Wessendorf and Elde (1986). Briefly, rehydrated sections were incubated in $10 \%$ normal swine serum and then immunostained with combinations of primary antisera containing goat antiserum to serotonin paired with rabbit antiserum to somatostatin or rabbit antiserum to oxytocin. Immunostaining was detected using a combination of secondary antisera that contained rhodamine-labeled swine immunoglobulins to goat IgG and fluoresceinlabeled swine immunoglobulins to rabbit IgG. Sections were examined and photographed through the Zeiss microscope using reflected fluorescence illumination to examine immunohistochemical staining and transmitted dark-field UV illumination to examine True blue or FluoroGold fluorescence.

Sections processed in this manner were also evaluated quantitatively to determine whether these populations of retrogradely labeled neurons could be distinguished by virtue of the immunostained fibers and terminals that appeared to appose labeled somata. Specifically, we counted preganglionic sympathetic neurons retrogradely labeled from the cervical sympathetic trunk and from the adrenal medulla in horizontal sections of spinal cord sampled from segmental levels C8-T3 and T7$\mathrm{T} 10$, respectively. These cells were classified either as appearing to be apposed or as not apposed by fibers or terminals immunostained for the particular neurotransmitter in question. For the purposes of this study, an apposition was defined as occurring when no distance was perceptible between a labeled cell and a labeled varicosity when using a $40 \times, 0.9$ numerical aperature objective. If a retrogradely labeled cell appeared to be apposed by 3 or more immunoreactive varicosities, or if its profile was outlined or traversed by an immunoreactive fiber, we classified that cell as apposed. Cells that failed to meet these criteria were classified as either not apposed or uncertain. To insure against classifying structures in 2 different planes as apposed, we did not refocus the microscope between observations of the tissue under UV and fluorescence illumination. Contingency tables $(2 \times 2)$ were generated and the difference between observed and expected numbers of labeled neurons apposed by immunostained fibers or varicosities was compared, using the chi-square statistic (Snedecor and Cochran, 1967).

The primary antisera used in these studies were goat antiserum to serotonin (43H2TG; ImmunoNuclear, Stillwater, MN), rabbit antiserum to somatostatin 15-28 (Maley and Elde, 1982) and rabbit antiserum to oxytocin (R111G; generated in this laboratory). They were used at a dilution of 1:100. All antisera were diluted with $0.05 \mathrm{M}$ PBS, pH 7.2, containing $0.3 \%$ (vol/vol) Triton X-100 (Sigma, St. Louis, MO). The specificity and sensitivity of these primary antisera were established using liquid-phase absorption controls with homologous and heterologous antigens and the solid-phase immunoblot technique of Larsson (1981).

The secondary antisera used in these studies were lissamine rhoda- 
mine sulfonyl chloride-labeled swine immunoglobulins to goat IgG (6301; Tago, Burlingame, $\mathrm{CA}$ ) and fluorescein isothiocyanate-labeled swine immunoglobulins to rabbit IgG (F205; Dako, Santa Barbara, CA). They were used at a dilution of 1:10. These antisera do not stain rat brain in the absence of primary antisera and appear not to cross-react with rabbit IgG or goat IgG, respectively (Wessendorf and Elde, 1986).

Since the somatostatin and oxytocin antisera were used in combination with the scrotonin antiserum, additional controls for specificity were performed. The hypothalamic median eminence, an area of the brain rich in both somatostatin and oxytocin immunoreactivity, was immunostained for serotonin in combination with either somatostatin or oxytocin. Under blue illumination, intense green fluorescence was noted in the zona externa of median eminence when immunostained with the antisera to somatostatin or oxytocin in combination with the antiserum to serotonin. When the illumination was switched to green light, we noted only sparse red fibers or terminals representing serotoninlike immunoreactivity. Thus it was concluded that the antiserum directed against serotonin was not cross-reacting with somatostatin- or oxytocin-like immunoreactivity or interacting with these primary antibodies. In spinal cord immunostained with these combinations of antisera, no structures were observed that displayed both red and green fluorescence. Thus, it appeared that antisera directed against somatostatin and oxytocin were not cross-reacting with serotonin-like immunoreactivity, nor were they interacting with the serotonin antiserum.

\section{Results}

CST neurons, retrogradely labeled with Fluoro-Gold or True bluc, were detected with UV illumination in the IML ipsilateral to the site of dye application (cut cervical sympathetic trunk). They were observed between spinal levels C8 and T5, but the majority of labeled cell bodics occurred between spinal levels T1 and T3 (Fig. 1a). Their frequency and distribution corresponded with that reported by Rando et al. (1981). Similarly, SAP neurons, retrogradely labeled with True blue or FluoroGold, were detected with UV illumination. They too were located ipsilateral to the site of dye administration (injected adrenal gland). They were obscrved between spinal levels $\mathrm{T} 1$ and L2, but the majority of labeled somata occurred between spinal levels $\mathrm{T} 7$ and T10 (Fig. $1 \mathrm{~b}$ ). Their frequency and pattern of distribution also corresponded to those previously reported (Schramm et al., 1975; Haase et al., 1982; Holets and Elde, 1982). The pattern and distribution of retrogradely labeled CST or SAP neurons were independent of whether they were retrogradely labeled with Flouro-Gold or with True bluc.

Simultaneous retrograde labeling of CST and SAP neurons with contrasting fluorescent dyes revealed that thesc 2 groups of preganglionic sympathetic neurons overlapped in limited segments containing the IML. This was especially evident between levels T3 and T5. Moreover, in areas of IML where these 2 groups of retrogradely labeled neurons overlapped, SAP neurons most often occupied the most lateral aspect of the nucleus (Fig. $1, c, d)$.

Further experiments were performed to establish that these 2 groups of neurons, retrogradely labeled from different sites, did not send collaterals to both sites of dye application. Using the techniques described, CST and SAP neurons were retrogradely labeled simultaneously with either Diamidino yellow or True blue. Diamidino yellow stains the nuclei of retrogradely labeled cells yellow (Keizer et al., 1983). Under these conditions, a single neuron with collaterals both to the supcrior cervical ganglion and the adrenal medulla would appear through the microscope as a cell with blue fluorescing cytoplasm and a yellow nucleus, using UV illumination (Keizer et al., 1983; Skirboll et al., 1984). Although many cells were intensely single-labeled in IML with one or the other tracer dye, no double-labeled ncurons were ever detected in the spinal cords of rats receiving this treatment. This was the case regardless of whether Diamidino yellow was applied to the cut CST and True blue was infused into the adrenal medulla or vice versa. Thus, it appeared that these 2 groups of retrogradely labeled neurons represented distinct, target-specific subnuclei of IML. Consistent with the pattern of staining observed when Fluoro-Gold and True blue were the retrograde dyes, in areas where True blue (CST) and Diamidino yellow (SAP) neurons overlapped, Diamidino yellow-labeled neurons occupied the more lateral aspects of IML.

Studies were next undertaken to determine whether these 2 target-specific subpopulations of neurons within IML were recipients of ncurochemically distinct afferents. As reported previously, some fibers and terminals in IMI, that displayed somatostatin-like immunoreactivity appeared to appose retrogradely labeled SAP neurons (Holets and Elde, 1982). In addition, we also noted varicosities that displaycd somatostatinlike immunoreactivity apposed to retrogradely labeled CST neurons (Fig. 2, $a, b$ ). Similarly, many fibers and terminals in IML that displayed serotonin-like immunoreactivity appeared to appose retrogradely labeled SAP neurons, as has been described (Holets and Elde, 1982; Appel et al., 1986). We also noted varicosities immunoreactive for serotonin in apposition to retrogradely labeled CST neurons (Fig. 2, e,f). This pattern of staining was not evident with oxytocin, however. Oxytocinimmunoreactive varicosities were detected that appeared to appose retrogradely labcled CST ncurons (Fig. 2, c, d). On the other hand, out of 66 retrogradcly labeled SAP neurons classified, we observed only one profile that appeared to be apposed by an oxytocin-immunoreactive structure. In this instance, the apposition appeared to be occurring on the proximal dendrite of the labeled soma. Using the chi-square statistic, a significant difference $(p<0.005)$ between the number of retrogradely labeled CST neurons that appeared to be apposed by oxytocinimmunoreactive varicosities and the number of retrogradely labeled SAP neurons was revealed. No significant difference $(p>0.10)$ was noted between the 2 populations of retrogradely labeled neurons when the basis for classification was apposition by either somatostatin-or serotonin-immunoreactive structures (Fig. 3).

\section{Discussion}

The sympathetic division of the autonomic nervous system is organized in a hierarchical arrangement. Thus, for independent regulation of specific targets of the sympathetic outflow there must be a specific final common pathway via which activation of a specific target can be regulated. Discrete populations of preganglionic sympathetic neurons innervating discrete populations of postganglionic sympathetic neurons would be such a pathway. The results of the retrograde tracing experiments in this study provide support for such an arrangement. That is, there are at least 2 target-specific populations of preganglionic sympathetic neurons forming subnuclei of the IML in the rat. One of these subnuclei contains preganglionic sympathetic neurons that project to the adrenal medulla. The other identified subnucleus contains preganglionic sympathetic neurons that project to a sympathetic chain ganglion, the superior cervical ganglion. This arrangement of preganglionic sympathetic neurons in IML is noteworthy, in that it suggests an anatomical substrate by which differential regulation of sympathetic outflow in general, and catecholamine secretion in particular, may be mediated. In rats and humans the adrenal medulla is the site from which plasma epinephrine, as well as some norepinephrine 

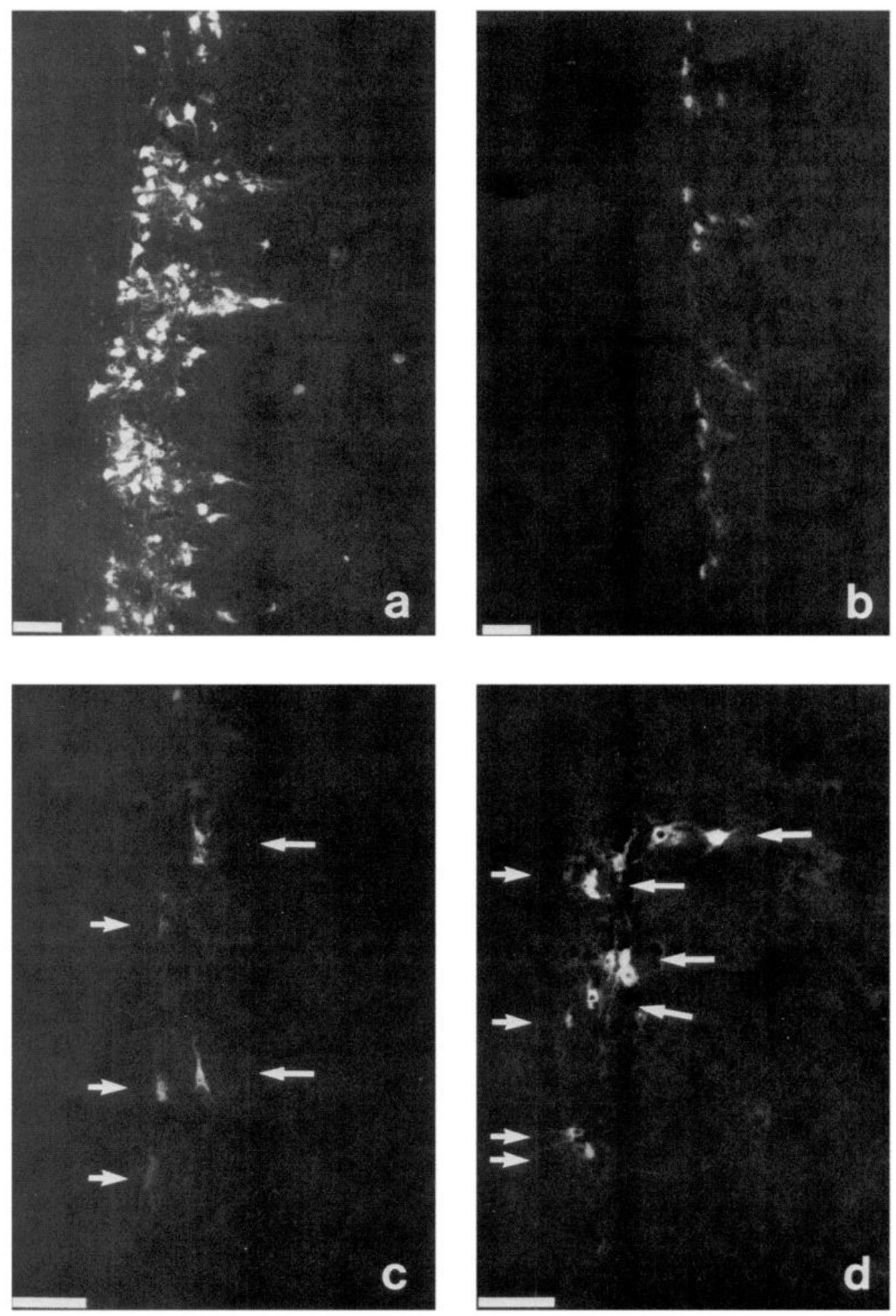

Figure 1. $a-d$, Fluorescence photomicrographs of uncoverslipped horizontal sections through rat IML. They were illuminated with dark-field UV light. $a$, CST neurons at T2 retrogradely labeled with Fluoro-Gold. $b$, SAP neurons at T9 retrogradely labeled with True blue. $c$, Overlapping SAP and CST neurons in the IML pars principalis at T4 retrogradely labeled with True blue and Fluoro-Gold, respectively. SAP neurons (small arrows), appeared blue through the microscope. CST neurons (larger arrows), appeared gold through the microscope. $d$, Overlapping SAP and CST neurons in the IML pars principalis and intercalatus proprius at T3, retrogradely labeled with True blue and Fluoro-Gold, respectively. SAP neurons (small arrows), appeared blue through the microscope. CST neurons (larger arrows), appeared gold through the microscope. Scale bar, $100 \mu \mathrm{m}$.

(about 30\% in rats), is secreted into the circulation (Price, 1957; Kvetnansky et al., 1979; Yamaguchi and Kopin, 1979). Overflow from vascular neuroeffector junctions of postganglionic sympathetic neurons, whose somata reside in sympathetic gan- glia, such as the superior cervical, is the principal source of circulating norepinephrine (Price, 1957; Yamaguchi and Kopin, 1979; Kopin et al., 1984).

Early theories suggested that the entire sympathetic nervous 

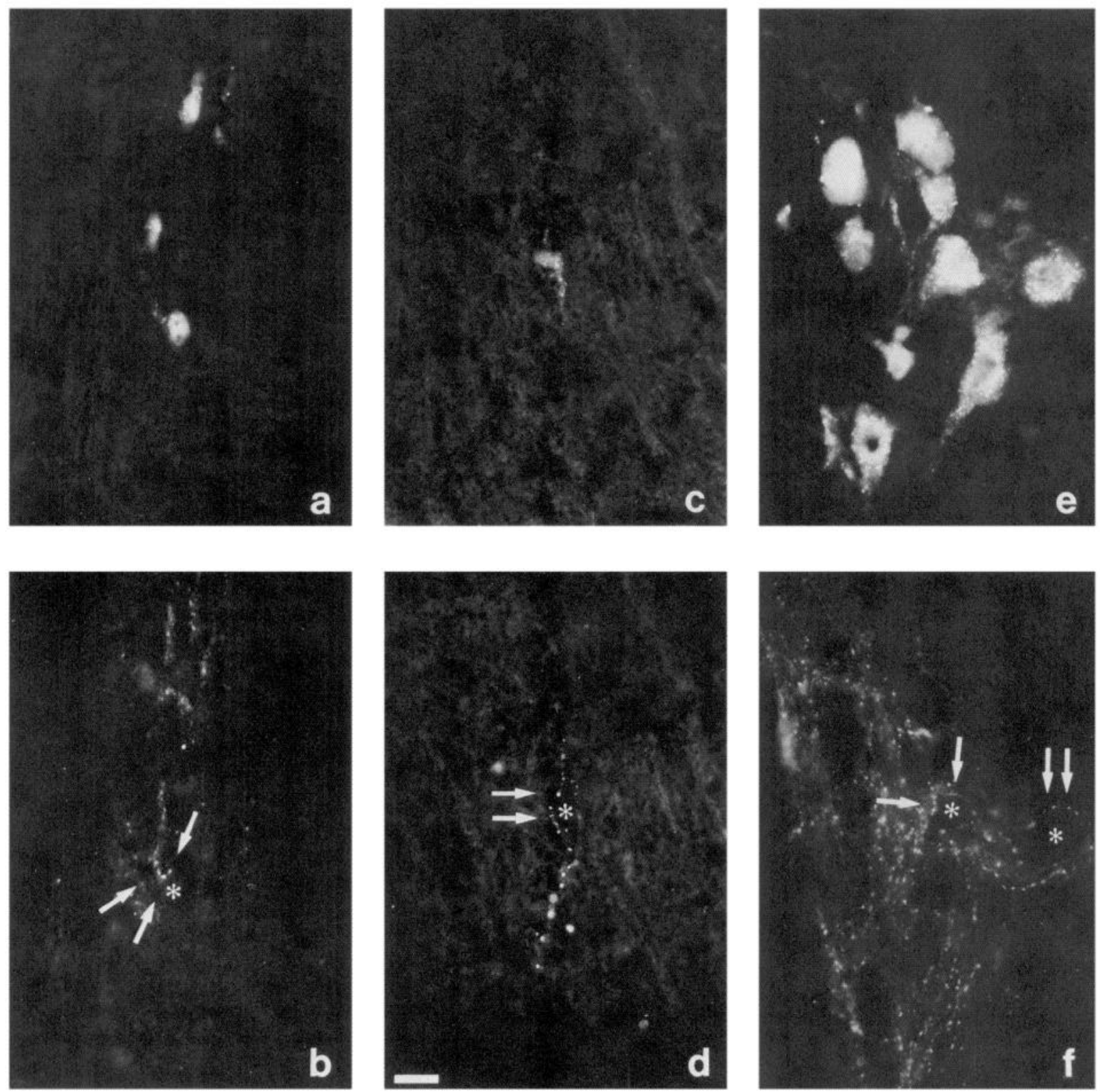

Figure 2. $a-f$, Photomicrographs of horizontal sections through IML in thoracic spinal cord. They were viewed with either dark-field UV ( $a$, $c$, $e$ ) or reflected fluorescence $(b, d, f)$ illumination. $a, b$, Section through T2. CST neurons retrogradely labeled with Fluoro-Gold are depicted in $a$. $b$, The same field was illuminated with reflected blue light to detect somatostatin-like immunoreactivity. Somatostatin-immunoreactive terminals appearing to appose a retrogradely labeled CST neuron are indicated by arrows. $c, d$, Section through T1. A CST neuron retrogradely labeled with Fluoro-Gold is depicted in $c$. $d$, The same field was illuminated with reflected blue light to detect oxytocin-like immunoreactivity. Oxytocinimmunoreactive terminals appearing to appose this retrogradely labeled CST neuron are indicated by arrows. $e$, $f$, Section through T2. CST neurons retrogradely labeled with Fluoro-Gold are depicted in $e$. $f$. The same field was illuminated with reflected green light to detect serotonin-like immunoreactivity. Serotonin-immunoreactive terminals appearing to appose a retrogradely labeled CST neuron are indicated by arrows. Asterisks denote the location of retrogradely labeled fluorescent somata with respect to immunofluorescent varicosities. Scale bar, $25 \mu \mathrm{m}$.

system functioned as a unit. Thus the level of activation of the entire sympathetic outflow increased or decreased as a whole (Cannon and Rosenblueth, 1937). However, evidence has been presented that suggests there is selective regional control of sympathetic outflow to specific targets or organs. In rabbits, electrical stimulation of different hypothalamic areas can result in parallel as well as opposite changes in simultaneous cardiac and renal sympathetic nerve activity (Okada and Ninomiya, 1983). Plasma catecholamine secretion can also be used as an index of sympathetic nervous system activity (Yamaguchi and Kopin, 1979; Kopin et al., 1984). Using this criterion, a number of neuroactive substances have been demonstrated as increasing central sympathetic outflow in rats. Bombesin, $\beta$-endorphin, and corticotropin-releasing factor all increase plasma catecholamine concentration following central nervous system administration in rats. However, while bombesin and $\beta$-endorphin are more potent at stimulating epinephrine secretion than norepinephrine secretion, corticotropin-releasing factor is a more potent stimulus for norepinephrine secretion (Van Loon et al., 1981; Brown and Fisher, 1984). Similarly, it has been demonstrated in cats that focal electrical stimulation of discrete but adjacent brain sites may have different effects on systemic plas- 
ma norepinephrine concentration, as compared to epinephrine concentration (Stoddard-Apter et al., 1983), although this response may also reflect differential secretion of norepinephrine versus epinephrine directly from the adrenal medulla (Matsui, 1984; Katafuchi et al., 1986). There is also evidence for regional regulation of sympathetic outflow in humans. Hyperventilation or the performance of mental arithmetic decreases sympathetic nerve activity in muscle, but increases sympathetic nerve activity to skin (Delius et al., 1972). Syncope causes large changes in plasma epinephrine secretion but has only a small effect on plasma norepinephrine. On the other hand, treadmill exercise induces similar changes in the magnitude of plasma epinephrine and norepinephrine (Robertson et al., 1979).

Previous studies have suggested that there are populations of preganglionic sympathetic neurons in IML projecting to specific targets. Dalsgaard and Elfvin $(1979,1981)$ used horseradish peroxidase to retrogradely label preganglionic sympathetic neurons projecting to either the superior cervical, stellate, or inferior mesenteric ganglia in the guinea pig. The majority of neurons retrogradely labeled from these sites were observed between spinal levels $\mathrm{T} 2$ and $\mathrm{T} 4, \mathrm{~T} 2$ and $\mathrm{T} 5$, and $\mathrm{L} 2$ and $\mathrm{L} 3$, respectively. However, since only a single retrograde transport marker was used, it could not be concluded from these studies whether labeled cell bodies had collateral projections to other targets or were, in fact, overlapping subnuclei of IML with target-specific projections. Subsequently, Hancock (1982) used horseradish peroxidase and DAPI to accomplish simultaneous retrograde labeling of preganglionic sympathetic neurons from the central cut ends of the intermesenteric and caudal lumbar sympathetic trunks in golden hamsters. Two distinct subgroups of retrogradely labeled preganglionic neurons were revealed, with similar segmental distributions between T12 and L3. However, cells labeled from the intermesenteric trunk were observed bilaterally in IML and in the dorsal commissural nuclcus, whilc cclls rctrogradely labeled from the lumbar sympathetic trunk were observed primarily in the ipsilateral IML. In the present study, retrogradely labeled cells were also observed dorsal to the central canal, but retrogradely labeled cells in the pars principalis of IML were only noted ipsilateral to the site of dye administration. Also in contrast to the above study, where retrogradely labeled CST and SAP neurons overlapped, SAP neurons were positioned more laterally. The results of the 2 studies are in agreement in that no double-labeled cells were observed. Most recently, Forehand and Rubin (1986) simultaneously applied True blue and Diamidino yellow to cut rostral and caudal ends of individual ganglia in the sympathetic chain. They did observe some double-labeled neurons $(<2 \%)$ in midthoracic segments. However, they concluded that individual preganglionic neurons project axons either rostral or caudal from single segments, but not both. Thesc studies lend support to the hypothesis that different populations of preganglionic sympathetic neurons may form a substrate for differential control of sympathetic outflow.

Another requirement for independent regulation of sympathetic outflow to individual targets is that discrete populations of neurons from "other brain centers" innervate discrete populations of preganglionic sympathetic neurons. However, in addition to specificity of origin and destination, there is another criterion that, if satisfied, destines that that population of neurons will be discrete. A subpopulation of neurons originating from a given nucleus can also be considered discrete if it is neurochemically distinct from other neurons in that nucleus. Thus, immunohistochemistry was used in the present study to investigate the possibility that such neurochemically distinct

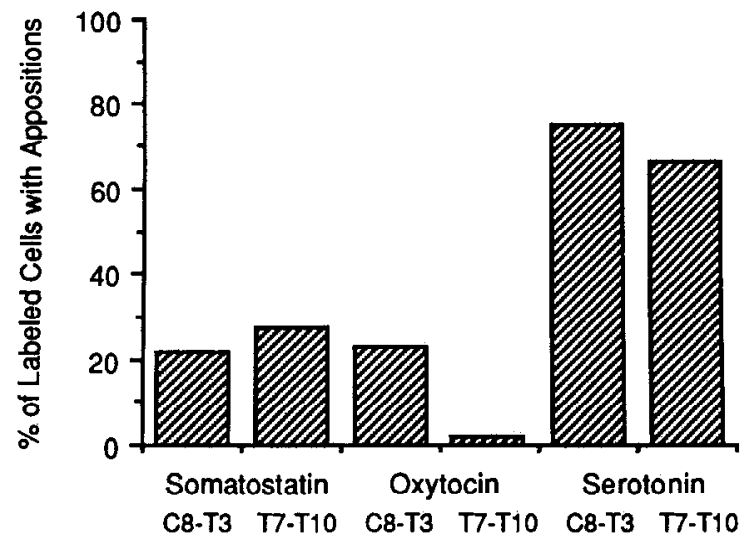

Figure 3. Comparison of the percentage of retrogradely labeled CST neurons and SAP neurons, counted between spinal levels C8 and T3 and $T 7$ and $T 10$, respectively, appearing apposed by somatostatin-, oxytocin-, or serotonin-immunoreactive fibers or varicosities. A total of 83,68 , and 149 retrogradely labeled CST neurons were observed in tissues immunostained for somatostatin, oxytocin, or serotonin respectively. We were unsure of how to classify 5,1 , and 5 cells in the each of the respective immunostaining groups. We also observed 68,66 , and 134 retrogradely labeled SAP neurons in tissues immunostained for somatostatin, oxytocin, or serotonin, respectively. We were unsure of how to classify 3,4 , and 9 cells in each of the respective immunostaining groups. The difference between expected and actual numbers of oxytocin-apposed CST neurons and SAP neurons was statistically significant $\left(\chi^{2}=14.1 ; p<0.005\right)$. The criteria for classifying a retrogradely labeled neuron as apposed were described in Materials and Methods.

populations of neurons might differentially innervate distinct populations of preganglionic sympathetic neurons in IML.

Previously, Tucker and Saper (1985) used retrograde labeling to demonstrate that the descending innervation of sympathetic preganglionic neurons to adjacent spinal segments was independent, but they could not discern a topographic organization of neurons innervating specific spinal levels. They did not investigate the neurochemical identity of this innervation. On the other hand, Holets and Elde (1982) observed that somatostatinlike immunoreactive fibers and terminals in IML appeared to appose retrogradely labeled SAP neurons preferentially within the segments examined. There is also physiological evidence suggesting a role for somatostatin in mediating differential activation of the sympathetic outflow. Intracerebroventricular administration of somatostatin inhibited adrenal and superior laryngeal nerve activity, but had no effect on firing rate in the CST (Somiya and Tonoue, 1984; Tonoue et al., 1985). Moreover, central nervous system administration of somatostatin, or a somatostatin analog, blunted epinephrine secretion in response to simultancously administered neuroactive peptides, but had little or no effect on norepinephrine secretion in responses to these peptides (Fisher and Brown, 1980; Van Loon et al., 1981; Brown and Fisher, 1984). In contrast to somatostatin, oxytocin-like immunoreactivity, also present in IML (Swanson and McKellar, 1979), was not found in proximity to retrogradely labeled SAP neurons in rat (Holets and Elde, 1982). In cat, the nonuniform distribution of somatostatin- and oxytocin-immunoreactive fibers and terminals in IML prompted investigators to speculate that these substances may have organspecific functions (Krukoff et al., 1985).

On the basis of these reports, we examined the 2 populations of preganglionic neurons we had identified to see whether they could be distinguished by appearing to be apposed by either 
somatostatin- or oxytocin-immunoreactive afferents. For comparison, we also examined serotonin-like immunoreactivity in this context. Serotonin-immunoreactive varicosities have been reported to appose retrogradely labeled SAP neurons as well as many unlabeled neurons in IML (Holets and Elde, 1982; Appel et al., 1986). In addition, significant numbers of scrotonin-immunoreactive fibers and terminals have been demonstrated in rat IML at levels of the thoracic spinal cord corresponding to the sites where the greatest numbers of retrogradely labeled CST and SAP neurons have been identified (Steinbusch, 1981; Appel et al., 1987). While it is recognized that the appearance of appositions to labeled cell bodies may not constitute bona fide synapses and that the level of resolution of the present technique does not rule out the possibility of axodendritic interaction, the appearance of such appositions would suggest the possibility of a functional relationship.

The present data did not support the hypothesis that these target-specific and functionally different subnuclei of IML could be differentiated by whether their neurons appeared to be apposed by somatostatin-immunoreactive afferents $(p>0.10$; Fig. $3)$. This was surprising in view of the anatomical and physiological data that appeared to support this hypothesis (see above). These data do not rule out the possibility that somatostatin is indeed acting in IML to differentiate these subnuclei. Two different forms of somatostatin have been immunohistochemically identified in spinal cord (Ho and Berelowitz, 1984). Thus, the possibility exists that the 2 identified subnuclei are regulated by neurons expressing different forms of somatostatin. Somatostatin-immunoreactive fibers in IML may also be apposing CST preganglionic neurons that stimulate postganglionic neurons in the superior cervical ganglion, which uses a substance other than norepinephrine as its predominant neurotransmitter (Heym and Lang, 1986).

Oxytocin-immunoreactive fibers and terminals appeared to avoid SAP neurons selectively as compared to CST neurons. However, oxytocin-immunoreactive fibers and terminals were present in other portions of IML at segmental levels where retrogradely labeled SAP neurons were visible. Thus it would appear that the paucity of oxytocin-immunoreactive afferents to SAP neurons is not a reflection of a lack of oxytocin-immunoreactive fibers and terminals at these segmental levels. These data suggest that oxytocin may be a neurotransmitter by which differential regulation of central sympathetic outflow is mediated. It has been reported that oxytocin-immunoreactive fibers appear to innervate IML preferentially between levels T1 and T3, T9 and T11, and T13 and L2 in rat (Swanson and McKellar, 1979). In the rat, the majority of SAP neurons are found between spinal segments T9 and T11 (Schramm et al., 1975; Haase et al., 1982; Holets and Elde, 1982). Thus, if SAP neurons are innervated by descending projections from the paraventricular nucleus, these fibers would represent a population of paraventricular neurons that does not use oxytocin as a neurotransmitter. The supraoptic nucleus, the other major brain site of oxytocin synthesis, does not project to spinal cord (for review, see Swanson and Sawchenko, 1983). Segments T1-T3 are the levels of rat spinal cord in which the majority of CST neurons were observed in this study and others (Rando et al., 1981). However, physiological evidence in support of the hypothesis that oxytocin may mediate differential regulation of sympathetic outflow is scant. In rats, intrathecal oxytocin incrcases heart rate and this effect is greater when administered at T2 than at T9 (Yashpal et al., 1987). However, microion- tophoretic application of oxytocin to CST neurons in rats stimulated only 1 of 23 antidromically identified units, while inhibiting 16 (Gilbey et al., 1982). On the other hand, in cats both oxytocin and vasopressin had excitatory effects on sympathetic preganglionic neurons projecting to the stellate ganglion (Backman and Henry, 1984). Others have suggested that the effects of intrathecal oxytocin in increasing blood pressure and decreasing urine flow are due to oxytocin's acting at vasopressin receptors (Riphagen and Pittman, 1986). Clearly, further physiological studies are necessary to resolve this issue.

Serotonin-immunoreactive fibers and terminals did not appear to have a preferential relation with either of the 2 identified groups of preganglionic sympathetic neurons. This finding was consistent with the observations of others, who reported significant serotonin-like immunoreactivity in rat IML at levels of the thoracic spinal cord corresponding to the site where the greatest numbers of retrogradely labeled CST and SAP neurons have been identified (Steinbusch, 1981; Appel et al., 1987). Moreover, Holets and Elde (1982) observed that, in addition to SAP neurons, serotonin-immunoreactive fibers were also afferent to other unidentified neurons in IML. The role of serotonin in regulating central sympathetic outflow is controversial. Some investigators have reported that serotonin increases sympathetic nerve-firing frequency following microiontophoretic application in IML (De Groat and Ryall, 1967; Coote et al., 1981; McCall, 1983). Others have reported that serotonin and its precursor 5-hydroxytryprophan inhibit spontaneous and elicit reflex preganglionic sympathetic nerve activity (Hare et al., 1972; Coote and MacLeod, 1974; Felpel and Huffman, 1982). In any case, it appears that serotonin-immunoreactive fibers and terminals are ubiquitous in rat IML.

To summarize this report, we have employed simultaneous retrograde tract-tracing techniques to demonstrate that there are at least 2 target-specific subnuclei in rat IML comprised of preganglionic sympathetic neurons. One of these subnuclei projects to the adrenal medulla, the site from which plasma epinephrine and some norepinephrine are secreted into the circulation. The other identified subnucleus projects to the superior cervical ganglion. When stimulated, synaptic overflow from terminals of postganglionic neurons arising from this ganglion presumably contribute in part to increased plasma norepinephrine. Furthermore, we used immunohistochemical techniques to reveal that, in addition to their target specificity, these subnuclei may be distinguished by virtue of the neurochemical identity of their afferent innervation. These data appear to be in concert with observations from 2 other rat brain nuclei, the paraventricular nucleus and nucleus tractus solitarius. Both of these bilateral nuclei are comprised of target-specific subnuclei and play important roles in autonomic nervous system function (Sawchenko and Swanson, 1982; Kalia et al., 1984). It is concluded from the present study that the IML and its afferent input may provide an anatomical substrate through which differential control of sympathetic nervous system activity is mediated.

\section{References}

Appel, N. M., M. W. Wessendorf, and R. P. Elde (1986) Coexistence of serotonin- and substance P-like immunorcactivity in nerve fibers apposing identified sympathoadrenal preganglionic neurons in rat intermediolateral cell column. Neurosci. Lett. 65: 241-246.

Appel, N. M., M. W. Wessendorf, and R. P. Elde (1987) Thyrotropinreleasing hormone in spinal cord: Coexistence with serotonin and 
with substance $P$ in fibers and terminals apposing identified preganglionic sympathetic neurons. Brain Res. 415: 137-143.

Appenzeller, O. (1982) The Autonomic Nervous System, pp. 1-30, Elsevier, Amsterdam.

Backman, S. B., and J. L. Henry (1984) Effects of oxytocin and vasopressin on thoracic sympathetic preganglionic neurones in the cat. Brain Res. Bull. 13: 679-684.

Brown, M. R., and L. A. Fisher (1984) Brain peptide regulation of adrenal epinephrine secretion. Am. J. Physiol. 247: E41-E46.

Cannon, W. B., and A. Rosenblueth (1937) Autonomic Neuro-Effector Systems, Macmillan, New York.

Coote, J. H., and V. H. MacLeod (1974) The influence of bulbospinal monoaminergic pathways on sympathetic nerve activity. J. Physiol. (Lond.) 241: 453-475.

Coote, J. H., V. H. MacLeod, S. Fleetwood-Walker, and M. P. Gilbey (1981) The response of individual sympathetic neurones to microiontophoretically applied monoamines. Brain Res. 215 : 135-145.

Dalsgaard, C.-J., and L.-G. Elfvin (1979) Spinal origin of preganglionic fibers projecting onto the superior cervical ganglion and inferior mesenteric ganglion of the guinea pig, as demonstrated by the horseradish peroxidase technique. Brain Res. 172: 139-143.

Dalsgaard, C.-J., and L.-G. Elfvin (1981) The distribution of sympathetic preganglionic neurons projecting onto the stellate ganglion of the guinea pig. A horseradish peroxidase study. J. Auton. Nerv. Syst. 4: 327-337.

De Groat, W. C., and R. W. Ryall (1967) Excitatory action of 5-hydroxytryptamine on sympathetic preganglionic neurones. Exp. Brain Res. 3: 299-305.

Delius, W., K.-E. Hagbarth, A. Hongell, and B. B. Wallin (1972) Manoeuvres affecting sympathetic outflow in human skin nerves. Acta Physiol. Scand. 84: 177-186.

Erichsen, T. J., A. Reiner, and H. J. Karten (1982) Co-occurrence of substance P-like and Leu-enkephalin-like immunoreactivities in neurones and fibres of avian nervous system. Nature 295: 407-410.

Fclpcl, L. P., and R. D. Huffman (1982) Supersensitivity to norepinephrine and serotonin in the intermediolateral cell column following chronic spinal transection. Soc. Neurosci. Abstr. 8: 998.

Fisher, D. A., and M. R. Brown (1980) Somatostatin analog: Plasma catecholamine suppression mediated by the central nervous system. Endocrinology 107: 714-718.

Forehand, C. J., and E. Kubin (1986) Specificity of sympathetic preganglionic projections: Rat preganglionic neurons project to either rostral or caudal ganglia along the sympathetic chain. Soc. Neurosci. Abstr. 12: 1056.

Gilbey, M. P., J. H. Coote, S. Fleetwood-Walker, and D. F. Peterson (1982) The influence of the paraventriculo-spinal pathway, and oxytocin and vasopressin on sympathetic preganglionic neurones. Brain Res. 251: 283-290.

Haase, P., $\Lambda$. Contestabile, and B. A. Flumerfelt (1982) Preganglionic innervation of the adrenal gland of the rat using horseradish peroxidase. Exp. Neurol. 78: 217-221.

Hancock, M. B. (1982) Separate populations of lumbar preganglionic neurons identified with the retrograde transport of horseradish peroxidase (HRP) and 4,6-diamidino-2-phenylindole (DAPI). J. Auton. Nerv. Syst. 5: 135-143.

Hare, B. D., R. J. Neumayr, and D. N. Franz (1972) Opposite effects of L-Dopa and 5-HTP on spinal sympathetic reflexes. Nature 239: 336-337.

Heym, C., and R. Lang (1986) Transmitters in sympathetic postganglionic neurons. In Neurohistochemistry: Modern Methods and Applications, P. Panula, H. Paivarinta, and S. Soinila, eds., pp. 493525, Liss, New York.

Ho, R. H., and M. Berelowitz (1984) Somatostatin $28_{1-14}$ immunoreactivity in primary afferent neurons of the rat spinal cord. Neurosci. Lett. 46: 161-166.

Holets, V., and R. Elde (1982) The differential distribution of serotonergic and peptidergic fibers to sympathoadrenal neurons in the intermediolateral cell column of the rat: A combined retrograde axonal transport and immunofluorescence study. Neuroscience $7: 1155-$ 1174.

Kalia, M., K. Fuxe, T. Hökfelt, O. Johansson, R. Lang, D. Ganten, C. Cuello, and L. Terenius (1984) Distribution of neuropeptide immunoreactive nerve terminals within subnuclei of the nucleus of the tractus solitarius of the rat. J. Comp. Neurol. 222: 409-444.

Katafuchi, T., H. Yoshimatsu, Y. Oomura, and A. Sato (1986) Re- sponses of adrenal catecholamine secretion to lateral hypothalamic stimulation and lesion in rats. Brain Res. 363: 141-144.

Keizer, K., H. G. J. M. Kuypers, A. M. Huisman, and O. Dann (1983) Diamidino Yellow dihydrochloride (DY.2HCl); a new fluorescent retrograde neuronal tracer, which migrates only very slowly out of the cell. Exp. Brain Res. 51: 179-191.

Kopin, I. J., Z. Zukowska-Grojec, M. A. Bayroh, and D. S. Goldstein (1984) Estimation of intrasynaptic norepinephrine concentrations at vascular neuroeffector junctions in vivo. Naunyn-Schmiedebergs Arch. Pharmacol. 325: 298-305.

Krukoff, T. L., J. Ciriello, and F. R. Caleresu (1985) Segmental distribution of peptide- and 5HT-like immunoreactivity in nerve terminals and tibers of the thoracolumbar sympathetic nuclei of the cat. J. Comp. Neurol. 240: 103-116.

Kvetnansky, R., V. R. Weise, N. B. Thoa, and I. J. Kopin (1979) Effects of chronic guanethidine treatment and adrenal medullectomy on plasma levels of catecholamines and corticosterone in forcibly immobilized rats. J. Pharmacol. Exp. Ther. 209: 287-291.

Larsson, L.-I. (1981) A novel immunocytochemical model system for specificity and sensitivity of antisera against multiple antigens. $J$. Histochem. Cytochem. 29: 408-410.

Maley, B., and R. Elde (1982) Immunohistochemical localization of putative neurotransmitters within the feline nucleus tractus solitarii. Neuroscience 7: 2469-2490.

Matsui, H. (1984) Adrenal medullary secretion in response to diencephalic stimulation in the rat. Neuroendocrinology 38: 164-168.

McCall, R. B. (1983) Serotonergic excitation of sympathetic preganglionic neurons: A microiontophoretic study. Brain Res. 289: 121127.

Okada, Y., and I. Ninomiya (1983) Different cardiac and renal inhibitory and excitatory areas in rabbit hypothalamus. Am. J. Physiol. 244: H832-H838.

Price, H. L. (1957) Circulating adrenaline and noradrenaline during diethyl ether anesthesia in man. Clin. Sci. 16:377-387.

Rando, T. A., C. W. Bowers, and R. E. Zigmond (1981) Localization of neurons in the rat spinal cord which project to the superior cervical ganglion. J. Comp. Neurol. 196: 73-83.

Riphagen, C. L., and Q. J. Pittman (1986) Oxytocin and [1-deamino, 8-D-arginine]-vasopressin (dDAVP): Intrathecal effects on blood pressure, heart rate and urine output. Brain Res. 374: 371-374.

Robertson, D., G. A. Johnson, K. M. Robertson, A. S. Nies, D. G. Shand, and J. A. Oats (1979) Comparative assessment of stimuli that release neuronal and adrenomedullary catecholamines in man. Circulation 59: 637-643.

Sawchenko, P. E., and L. W. Swanson (1982) Immunohistochemical identification of neurons in the paraventricular nucleus of the hypothalamus that project to the medulla or to the spinal cord in the rat. J. Comp. Neurol. 205: 260-272.

Schmued, L. C., and J. H. Fallon (1986) Fluoro-Gold: A new fluorescent retrograde axonal tracer with numerous unique properties. Brain Res. 377: 147-154.

Schramm, L. P., J. R. Adair, J. M. Stribling, and L. P. Gray (1975) Preganglionic innervation of the adrenal gland of the rat: A study using horseradish peroxidase. Exp. Neurol. 49: 540-553.

Skirboll, L., T. Hökfelt, G. Norell, O. Phillipson, H. G. J. M. Kuypers, M. Bentivoglio, C. E. Catsman-Berrevoets, T. J. Visser, H. Steinbusch, A. Verhofstad, A. C. Cuello, M. Goldstein, and M. Brownstein (1984) A method for specific transmitter identification of retrogradely labeled neurons: Immunofluorescence combined with fluorescence tracing. Brain Res. Rev. 8: 99-127.

Snedecor, G. W., and W. G. Cochran (1967) Statistical Methods, pp. 215-220, Iowa State U. P., Ames, IA.

Somiya, H., and T. Tonouc (1984) Neural peptides as central integrators of autonomic nerve activity: Effects of TRH, SRIF, VIP and bombesin on gastric and adrenal nerves. Reg. Pept. 9: 47-52.

Steinbusch, H. W. M. (1981) Distribution of serotonin-immunoreactivity in the central nervous system of the rat-cell bodies and terminals. Neuroscience $6: 557-618$.

Stephanini, M., C. De Martino, and L. Zaboni (1967) Fixation of ejaculated spermatozoa for electron microscopy. Nature 216:173174.

Stoddard-Apter, S. L., A. Siegel, and B. E. Levin (1983) Plasma catecholamine and cardiovascular responses following hypothalamic stimulation in the awake cat. J. Auton. Nerv. Syst. 8: 343-360.

Swanson, L. W., and S. McKellar (1979) The distribution of oxytocin- 
and neurophysin-stained fibers in the spinal cord of the rat and monkey. J. Comp. Neurol. 188: 87-106.

Swanson, L. W., and P. E. Sawchenko (1983) Hypothalamic integration: Organization of the paraventricular and supraoptic nuclei. Annu. Rev. Neurosci. 6: 269-324.

Tonoue, T., H. Hata, T. Ohnishi, N. Kido, and T. Nakamura (1985) Somatostatin and GABA correlate with cervical autonomic nerve activity. Regul. Pept. 10: 299-307.

Tucker, D. C., and C. B. Saper (1985) Specificity of spinal projections from hypothalamic and brainstem areas which innervate sympathetic preganglionic neurons. Brain Res. 360: 159-164.

Van Loon, G. R., N. M. Appel, and D. Ho (1981) $\beta$-Endorphininduced increase in plasma epinephrine, norepinephrine and dopa- mine in rats: Inhibition of adrenomedullary response by intracerebral somatostatin. Brain Res. 212: 207-214.

Wessendorf, M. W., and R. P. Elde (1986) Characterization of an immunofluorescence technique for the demonstration of coexisting neurotransmitters within nerve fibers and terminals. J. Histochem. Cytochem. 33: 984-994.

Yamaguchi, I., and I. J. Kopin (1979) Plasma catecholamine and blood pressure responses to sympathetic stimulation in pithed rats. Am. J. Physiol. 237: H305-H310.

Yashpal, K., S. Gauthier, and J. L. Henry (1987) Oxytocin administered intrathecally preferentially increases heart rate rather than arterial pressure in the rat. J. Auton. Nerve Syst. 20:167-178. 